\title{
The effect of cigarette type on anthropometrics and weight of PLWH
}

\begin{abstract}
Background: The effects of cigarette smoking on body weight have rendered disparate results. This study explores this relationship among people living with HIV (PLWH). This population has a high smoking prevalence and is experiencing a steady increase in obesity rate. Noteworthy, they are frequent users of mentholated cigarettes, a type of cigarette frequently marketed for its flavor and slimming effect.

Methods: We used the baseline information collected in our randomized clinical trial for smokers living with HIV. Smoking history was obtained using self-report surveys (e.g. Fagerström Test for Nicotine Dependence) and smoking status was confirmed weekly via expired breath carbon monoxide. Anthropometrics were obtained using standardized procedures, and covariates including sociodemographics, diet, exercise, and previous weight were collected. Logistic regressions were used to examine associations between overweight $\left(\mathrm{BMI}>25 \mathrm{~kg} / \mathrm{mg}^{2}\right)$ and obese $\left(\mathrm{BMI}>30 \mathrm{~kg} / \mathrm{m}^{2}\right)$ participants, smoking history, and cigarette type.
\end{abstract}

Results: At baseline, participants $(\mathrm{n}=200)$ were, on average, $51.7 \pm 6.7$ years old, and smoked an average of $14.9 \pm 5$ cigarettes/day. Only a third were eutrophic, and females were heavier than males $(31.2 \pm 8.6$ vs. $26.5 \pm 5.5, \mathrm{p}=.02)$. The odds of obesity were higher among individuals older than 60 years of age than in those less than $20(\mathrm{OR}=3.1$ 95\% CI: 1.2-8.0 p=0.001). PLWH who smoked menthol-flavored cigarettes had significantly higher BMI scores than non-menthol smokers (29.2 \pm 7.8 vs. $25.2 \pm 4.4$, $\mathrm{p}=.02)$. Likewise, smokers of mentholated cigarettes had increased odds of abdominal obesity ( $\mathrm{OR}=1.4,95 \%$; $\mathrm{CI}: 1-1.6, \mathrm{p}=.05)$, compared to their non-menthol counterparts.

Conclusion: Obesity is a prevalent problem in this population, particularly among females. Here we show that differences in body weight are not the result of differential activity level, rather it is associated with the use of mentholated cigarettes.
Volume 8 Issue 5 - 2018

\author{
Maria Jose Miguez,' Calonie Gray, ${ }^{2}$ Clery \\ Quiros,' Caroline Perez' \\ 'Florida International University, USA \\ 2REDI LLC, USA
}

Correspondence: Maria Jose Míguez, MD, PhD, Florida International University, School of Integrated Science and Humanity, I 200 SW $8^{\text {th }}$ St. Suite AHC4-355, Miami, FL 33199 , USA,Email mjmiguez@fiu.edu

Received: August 15, 2018| Published: October 02, 2018

Keywords: obesity, mentholated cigarettes, Central obesity, waist to hip ratio, smoking

Abbreviations: WHR, waist to hip ratio; PLWH, people living with HIV; BMI, body mass index.

\section{Introduction}

Relying on evidence that smokers tend to weigh less than nonsmokers, tobacco companies have used several strategies to take advantage of, and manipulate, people's concern with weight. ${ }^{1,2}$ Their goal became the addition of active agents to target "non-smokers who are more concerned with losing weight than with contracting respiratory or blood circulatory illnesses. [...] (Bates no. 2056159412). ${ }^{2}$ While initially focusing on appetite suppressants, later companies began considering "Specific Appetite Inducers". ${ }^{2}$ Documents indicated that they were experimenting with "special herbs or medications in a cigarette form as appetite stimulants or possibly for tension release". 2 The list included tartaric acid, 2-Acethylpyridine, catecholamine, menthol, mariolide, propylene glycol and reserpine. They were aware that appetite can be strongly influenced by both aromatic and taste characteristics of the compounds, and inquired about menthol. ${ }^{3}$ Note worthy, experts indicated that menthol had reached inconclusive effects. Yet, menthol's other characteristics (e.g. odor, taste, and flavor) let to its inclusion and marketing. ${ }^{2,4}$ Although several decades have passed since the introduction of mentholated cigarettes to the market, the burgeoning literature is notable for the scarcity of studies examining the plausible effect of mentholated cigarettes on weight.
A recent insurgence of concern over the safety of this additive, and its potential relationship with weight gain, has prompted further research. ${ }^{6}$

Equally important, the fear of weight gain often impacts readiness to quit smoking in high-risk populations, including people living with HIV. ${ }^{7,8}$ These segments of the population have

a) An excessively high prevalence of smoking

b) Frequent use of mentholated cigarettes, and

c) Greater body image concerns (e.g. shape, form, and size). ${ }^{7,9}$

Body dissatisfaction has been associated with lower interest and attempts to quit smoking, and with poor antiretroviral adherence. ${ }^{8,10}$ Our study aims to address this gap by examining the relationships between use of menthol-flavored cigarettes and body composition using data from our ongoing, randomized clinical trial. Understanding how these additives are associated with weight may help determine the cumulative health risk that current smokers may have as a function of the type of cigarette used.

\section{Methods}

\section{Design and setting}

The Patch Study is an ongoing, randomized clinical trial involving 
$\mathrm{HIV}+$ smokers who were motivated to quit. The trial has been ongoing since 2016 and is taking place in Miami, Florida. The aim is to recruit a total of 500 participants. The trial was powered to detect differences in biochemically verified 7-day point prevalence abstinence at 3,6, and 12 months of follow-up ( $80 \%$ power, type I error rate of $\alpha=5 \%$, assuming a $20 \%$ loss to follow-up).

Current analyses spanned from June 2016 to December 2017, for a total of 18 months of enrollment and follow-up.

Adults were eligible ifHIV status, smoking status, and willingness to quit were confirmed. For safety reasons, subjects were excluded if they had any contraindication to nicotine patches or gums, were involved in other smoking and/or drug cessation or weight control programs, or had comorbid conditions that limited their safe participation, such as the presence of psychotic or disabling psychiatric disorders. Written study materials, informed consent forms, and the study protocol were approved by Western IRB (WIRB). All procedures occurred at the University of Miami's Clinical Translational Research Site.

\section{Smoking surveys}

After giving informed consent, participants provided an exhaled breath carbon monoxide sample (Vitalograph; Lenexa, KS) for biochemical verification of smoking status. Then, subjects completed several standardized surveys to profile smoking history, including the number of cigarettes smoked per day and the history of tobacco use (cigarettes vs. cigars). This data, along with age of initiation and the total number of years smoking, enables estimation of cumulative exposure. To measure nicotine dependence, we selected the Fagerström Test for Nicotine Dependence (FTND) due to the strong literature evidencing validity, reliability, and its availability in English and Spanish. ${ }^{11}$ Participants were also asked about exposure to secondhand smoke (SHS), symptoms of lung disease, and personal/family history of respiratory conditions. Upon completion of the baseline visit, participants expired breath carbon monoxide samples were verified.

\section{Use of menthol-flavored cigarettes}

This variable was based on self-reports (yes/no) and the participant's preferred brands. Smokers were dichotomized as menthol users or non-menthol users.

\section{Anthropometric and nutritional intakes}

Participants' anthropometric measures were obtained at each visit. Body weight and height were measured to the nearest $0.1 \mathrm{~kg}$ after removal of shoes and outerwear using a calibrated balance. Weight and height were used to calculate body mass index (BMI; weight $[\mathrm{lbs}] /$ height $[\text { inches }]^{2} \times 703$ ). Participants were classified as thin if BMI was less than $18.5 \mathrm{~kg} / \mathrm{m}^{2}$, eutrophic if BMI was 18.5 to $24.9 \mathrm{~kg} / \mathrm{m}^{2}$, overweight if BMI was 25 to $29.9 \mathrm{~kg} / \mathrm{m}^{2}$, and obese if BMI was $30 \mathrm{~kg} /$ $\mathrm{m}^{2}$ or higher. ${ }^{12}$ Studies suggest that wait to hip ratio (WHR) is more accurate than BMI for predicting the risks of cardiovascular disease and premature death, so we obtained those measures. ${ }^{13}$ As per national guidelines, the waist circumference measurement was made at the top of the iliac crest after fasting overnight. ${ }^{14,15}$ The hip circumference measurement was obtained around the widest portion of the buttocks. In accordance with national guidelines, abdominal obesity was defined as a waist circumference greater than 102 centimeters ( 40 inches) in males and 88 centimeters ( 35 inches) in females. ${ }^{16}$

\section{Covariates}

Computerized questionnaires were used to obtain dietary intake (24 hour food recall), socio-demographics, and the medical chart, including a history of ART. ${ }^{17}$ Based on self-reports, subjects were categorized as African American, White non-Hispanic (Caucasian), or Hispanic. Age was stratified by 20 year gaps between groups (18 to 39,40 to 59 , or 60 or more years). Annual income was categorized as $\$ 0$ to $\$ 11,000, \$ 11,001$ to $\$ 20,000, \$ 20,001$ to $\$ 49,999$, or more than $\$ 50,000$. Education level was assigned a code between 1 and 16 , representing each year of schooling from elementary/middle school through college or vocational training.

\section{Statistical analysis}

All statistical analyses were conducted using SPSS 21.0 (SPSS Inc., Chicago, IL, USA). For all analyses, statistical significance was considered as a two-tailed p-value $<0.05$. Means, standard deviation and percentages were used to describe the characteristics of the study sample. Analysis of variance (ANOVA) was performed to test for significant differences in mean of anthropometric measurements and the variables of interest. Regression analyses were used to evaluate predictors of body mass index and any factors that were significantly associated with BMI was included in the final model.

\section{Results}

\section{Recruitment}

A total of 310 HIV infected smokers were eligible, and are currently being followed in a smoking cessation and health outcomes study conducted in Miami, Florida. Reflecting the current trend of the HIV epidemic, the mean age was above 50 years (51.7years) in both groups, however, the extremes of the age distribution were 23 and 69 years old. There were approximately equal numbers of male and female participants. The total sample includes 6\% Caucasian, 83\% AfricanAmerican, and $11 \%$ Hispanic, so we have a large representation of minorities. The male to female ratio was nearly one to one (Table 1). Income levels were significantly different. Findings are in line with prior reports indicating that smoking rates are significantly higher for persons living below the poverty line. ${ }^{18}$

Table I General Characteristics of the Study Population

\begin{tabular}{llll}
\hline & Menthol & Non-Menthol & p-Value \\
\hline Age & $51.2 \pm 8.8$ & $52.0 \pm 8.6$ & 0.59 \\
Male & $54 \%$ & $58 \%$ & \\
Female & $46 \%$ & $42 \%$ & 0.39 \\
African American & 88 & 51 & \\
Hispanic & 9 & 19 & 0 \\
Caucasian & 3 & 30 & \\
Income & & & \\
$<10,000$ & $79 \%$ & $60 \%$ & 0.03 \\
10,000-29,000 & $18 \%$ & $32 \%$ & \\
$30,000-79,000$ & $3 \%$ & $8 \%$ & 0.29 \\
Cigarettes per day & $14.1 \pm 10.4$ & $15.9 \pm 8.5$ & \\
\hline
\end{tabular}

a) Values are means \pm deviations or percentages.

b) Significant differences were found for age, race/ethnicity and income 


\section{Obesity and overweight prevalence and associated sociodemographics}

Only a third of the sample was eutrophic, and females were heavier than males ( $31.2 \pm 8.6$ vs. $26.5 \pm 5.5, \mathrm{p}=.02)$. The odds of obesity was higher among individuals older than 60 years of age than in those less than $20(\mathrm{OR}=3.195 \% \mathrm{CI}: 1.2-8.0 \mathrm{p}=0.001)$. As illustrated in Table 2 , overweight and obesity vary greatly between men and women, with women being disproportionately affected. Education status also differed across the groups with more years of education reported by the obese group. Of interest, neither age, income, nor race/ethnicity differed among the study groups.

Table 2 Group Characteristics by BMI

\begin{tabular}{llll}
\hline & Mentholated cigarettes & Non-Menthol & P value \\
\hline Waist & $38.15 \pm 7.4$ & $36.36 \pm 5.3$ & 0.05 \\
Hip & $41.59 \pm 6.2$ & $39.69 \pm 4.36$ & 0.06 \\
Triceps & $18.9 \pm 11.3$ & $16.39 \pm 8.6$ & 0.11 \\
Abdomen & $38.90 \pm 19.0$ & $32.2 \pm 15.38$ & 0.03
\end{tabular}

a) Values are means \pm deviations or percentages.

b) Significant values were found for waist, hip, and abdominal ratios

\section{Does smoking affect body mass index/ weight?}

In contrast to the popular perception that smokers are thinner, overall BMI scores were $28.8 \pm 8.8$, highlighting the profile of this at-risk population. Of concern, only a third of the sample had a eutrophic weight classification. The remaining subjects were overweight $(32 \%)$ or obese $(37 \%)$. Females were heavier than males $(31.2 \pm 8.6 \mathrm{vs} .26 .5 \pm 5.5, \mathrm{p}=.02)$. Older individuals ( $>60$ years of age) were also more likely to be obese than those under 40 years of age $(\mathrm{OR}=3.195 \%$ CI: $1.2-8.0 \mathrm{p}=0.001)$. Middle aged subjects were more likely to be overweight than their younger counterparts (1839 y OR: $2, \mathrm{p}=0.03$ ). Based on prior findings suggesting that heavy smokers tend to have greater body weight than eutrophic smokers, we dichotomized the sample by $<10$ cigarettes/day or $20>$ cigarettes/day, then analyzed weight and BMI. ${ }^{19}$ We could not identify a significant difference between the BMI groups.

\section{Weight by type of cigarette at baseline}

To better understand how smoking and body weight relate, it is crucial to account for the type of cigarette being used. Despite similar amounts of smoking, and exercise patterns, PLWH who smoked menthol-flavored cigarettes had significantly higher BMI values than non-menthol smokers $(29.2 \pm 7.8$ vs. $25.2 \pm 4.4, \mathrm{p}=.02)$. As depicted in Table 2, additional analyses indicated differences in several anthropometric measures.

\section{Final analysis}

The risk of abdominal obesity increased by $40 \%$ for menthol users $(\mathrm{O}=1.4,95 \%$; CI: $1-1.6, \mathrm{p}=05)$. The mean waist, hip, and abdominal circumference of the menthol smokers was significantly higher than the non-menthol smokers. In Table 3, multivariate regression analysis confirmed that the type of cigarettes smoked significantly predicted BMI. In the adjusted model, female sex was also a significant predictor of obesity.
Table 3 Final Regression Analyses

\begin{tabular}{lllll}
\hline & Coefficient & Std Error & $\mathbf{t}$ & P Value \\
\hline $\begin{array}{l}\text { Mentholated } \\
\text { Cigarettes }\end{array}$ & 3.74758 & 1.4489 & 2.59 & 0.01 \\
Sex & -5.0568 & 0.9201 & -5.5 & 0.000 \\
Education & .5984 & .2031 & 2.95 & 0.04 \\
Income & & & & \\
\$I0-29K & -0.51822 & 1.1324 & -0.95 & 0.648 \\
\$30-79 K & -0.89859 & 2.4313 & -0.24 & 0.712 \\
African American & -1.86652 & 1.9576 & -0.95 & 0.341 \\
Hispanic & -0.55907 & 2.366 & -0.24 & 0.813 \\
\hline
\end{tabular}

\section{Discussion}

The analyses uncovered several interesting findings. Although the common belief is that smokers are skinnier, over two-thirds of our smokers were either overweight or obese. This is in line with many researchers describing a direct relationship between smoking and weight gain..$^{20,21}$ In this regard, our cohort of smokers living with HIV had a sizable proportion of obese and overweight participants. However, our study extends the current literature by focusing on PLWH, and by including the type of cigarette in the analyses. The exclusion of this variable may account for some of the previously mentioned discrepancies in recent studies. ${ }^{1-23}$ Additionally, it is possible that those with lower rates of mentholated cigarettes find their sample to be skinnier, and the opposite might occur if the population is composed primarily of females and minorities, the primary consumers of mentholated cigarettes..$^{1,4}$

The hypothesis that smoking mentholated cigarettes adversely affects weight was confirmed, as smokers of mentholated cigarettes were significantly heavier than non-menthol smokers with HIV. Findings are of concern because obesity and smoking rates are sky rocketing among people living with HIV, and both are significant contributors to morbidity and mortality. ${ }^{24,25}$ Interestingly, the markers of abdominal obesity such as percent body fat, waist circumference, and hip circumference were also higher in smokers of menthol cigarettes. This entails an even higher health risk of metabolic syndrome, incidental cardiovascular disease, and type II diabetes. ${ }^{26} \mathrm{As}$ being female led to an increased risk of obesity in the study, the study results should serve as a targeted health message.

The mechanisms underlying our findings are not been elucidated, however various causal mechanisms may account for the effect of smoking on central obesity. First, smoking stimulates the sympathetic nervous system, leading to an increase of cortisol, a stress hormone. ${ }^{27,28}$ Abdominal fat deposition appears to be related to elevated levels of serum cortisol. ${ }^{29}$ Second, cigarette smoking is related to increased insulin resistance, which is associated with increases in abdominal fat deposition and diabetes..$^{30,31}$

Third, since the waist and hip circumferences are determined by an individual's proportions of android fat and gynoid fat, an hormonal imbalance could be the cause. Smoking has an anti-estrogenic effect, leading to a decrease fat metabolism and to fat accumulation. ${ }^{32,33}$ Finally, another possible reason is that smokers have distinct lifestyle characteristics, such as a higher likelihood of depressive moods and 
sleep impairments, which are risk factors for central obesity. ${ }^{34,35} \mathrm{In}$ contrast to prior studies showing that a clustering of smoking, obesity, and lower socioeconomic status exist, we did not replicate those results in this sample. ${ }^{36}$ However, those of lower socioeconomic strata were more likely to be smokers of mentholated cigarettes. ${ }^{4}$ We were also unable to confirm prior findings from a national cohort indicating that body leanness increased with the duration of smoking. ${ }^{37}$

This research has important clinical and public health implications, as mentholated cigarettes are the only flavored cigarettes that have not been banned from the market. But it should, as data suggests an association with obesity, which, at minimum, warrants more research. These findings need to be analyzed in light of several limitations. This is a cross-sectional analysis, and the data was derived only from people living with HIV. Despite this, we have observed a similar pattern among the general population..$^{20,21}$ As with all human data, causality is difficult to establish, but the replication of this observation from several previous studies increases confidence. Our anthropometric measures were carefully obtained following national protocols. ${ }^{14,15}$

\section{Acknowledgments}

None.

\section{Conflicts of interests}

The author declares that there is no conflict of interest.

\section{References}

1. Shang C, Chaloupka FJ, Fong GT, et al. Weight control belief and its impact on the effectiveness of tobacco control policies on quit attempts: findings from the ITC 4 Country Project. Tobacco control. 2015.

2. Gonseth S, Jacot-Sadowski I, Diethelm PA, et al. The tobacco industry's past role in weight control related to smoking. The European Journal of Public Healt. 2012;22(2):234-237.

3. Yin $\mathrm{W}$, Hewson L, Linforth $\mathrm{R}$, et al. Effects of aroma and taste, independently or in combination, on appetite sensation and subsequent food intake. Appetite. 2017;114:265-274.

4. Lee YO, Glantz SA. Menthol: putting the pieces together. Tobacco Control. 2017;20(Suppl 2):1-7.

5. Antonio AMM, Fagan P, Hamamura FD, et al. Menthol cigarette smoking and obesity in young adult daily smokers in Hawaii. Preventive Medicine Reports. 2015;2:946-952.

6. Stewart ST, Cutler DM, Rosen AB Forecasting the Effects of Obesity and Smoking on U.S. Life Expectancy. The New England journal of medicine. 2009;361(23):2252-2260.

7. Shirley D, Thibodeau L, Catz SL, et al. Cessation-Related Information, Motivation, and Behavioral Skills in Smokers Living with HIV. AIDS care. 2018;30(2):131-139.

8. Fingeret MC, Vidrine DJ, Arduino RC, et al. The Association Between Body Image and Smoking Cessation Among Individuals Living with HIV/AIDS. Body image. 2007;4(2):201-206.

9. Sharma A, Howard AA, Klein RS, et al. Body image in older men with or at-risk for HIV infection. AIDS care. 2007;19(2):235-241.

10. Blashill AJ, Wilson JM, Baker JS, et al. Assessing appearance-related disturbances in HIV-infected men who have sex with men (MSM): Psychometrics of the Body Change and Distress Questionnair. AIDS and Behavior. 2014;18(6):1075-1084.
11. Fagerström KO, Kunze M, Schoberberger R, et al. Nicotine dependence versus smoking prevalence: comparisons among countries and categories of smokers. Tobacco control. 2008;103(5):841-845.

12. Hellec B, Campbell-Scherer D, Michael Allan G. The skinny on BMI and mortality. Canadian Family Physician. 2015;61(11):970.

13. Elsayed EF, Tighiouart H, Weiner DE, et al. Waist Hip Ratio and Body Mass Index as Risk Factors for Cardiovascular Events in Chronic Kidney Disease. American journal of kidney diseases : the official journal of the National Kidney Foundation. 2018;52(1):49-57.

14. Bradlee ML, Singer MR, Qureshi MM, et al. Food group intake and central obesity among children and adolescents in the Third National Health and Nutrition Examination Survey (NHANES III). Public health nutrition. 2010;13(6):797-805.

15. NHLBI. Obesity Education Initiative, National Heart, Lung, Blood Institute, North American Association for the Study of Obesity, Expert Panel on the Identification, Treatment of Overweight, Obesity in Adults (US). The practical guide: identification, evaluation, and treatment of overweight and obesity in adults. The Institute. 2000.

16. Klein S, Allison DB, Heymsfield SB, et al. Waist circumference and cardiometabolic risk: a consensus statement from shaping America's health: Association for Weight Management and Obesity Prevention; NAASO, the Obesity Society; the American Society for Nutrition; and the American Diabetes Association. Obesity. 2007;85(5):1197-1202.

17. Karvetti RL, Knuts LR. Validity of the 24-hour dietary recall. Journal of the American Dietetic Association. 1985;85(11):1437-1442.

18. Garrett BE, Dube SR, Trosclair A. Centers for Disease Control and Prevention (CDC). Cigarette smoking - united states. MMWR Surveill Summ. 2011;60(1):109-113.

19. Lohse T, Rohrmann S, Bopp M, Faeh D. Heavy Smoking Is More Strongly Associated with General Unhealthy Lifestyle than Obesity and Underweight. PLoS ONE. 2016;11(2):e0148563.

20. Watanabe T, Tsujino I, Konno S, Association between smoking status and obesity in a nationwide survey of Japanese adults. PloS one. 2016 Mar 23;11(3):e0148926.

21. Tuovinen EL, Saarni SE, Männistö S, et al. Smoking status and abdominal obesity among normal-and overweight/obese adults: Population-based FINRISK study. 2016;4:324-330.

22. Clair C, Rigotti NA, Porneala B, et al. (Association of smoking cessation and weight change with cardiovascular disease among adults with and without diabetes. JAMA. 2013;309(10):1014-1021.

23. Lahti-Koski M, Pietinen P, Heliövaara M, et al. Associations of body mass index and obesity with physical activity, food choices, alcohol intake, and smoking. Am J Clin Nutr. 2002;75(5):809-817.

24. Guehi C, Badjé A, Gabillard D, et al. High prevalence of being Overweight and Obese HIV-infected persons, before and after 24 months on early ART in the ANRS 12136 Temprano Trial. AIDS Research and Therapy. 2016;13:12.

25. Mdege ND, Shah S, Ayo-Yusuf OA, et al. Tobacco use among people living with HIV: analysis of data from Demographic and Health Surveys from 28 low-income and middle-income countries. The Lancet Global Health. 2017;5(6):e578-e592.

26. Ginsberg HN, MacCallum PR. The Obesity, Metabolic Syndrome, and Type 2 Diabetes Mellitus Pandemic: Part I. Increased Cardiovascular Disease Risk and the Importance of Atherogenic Dyslipidemia in Persons With the Metabolic Syndrome and Type 2 Diabetes Mellitus. Journal of the cardiometabolic syndrome. 2009;4(2):113-119. 
27. Steptoe A, Ussher M. Smoking, cortisol and nicotine. International Journal of Psychophysiology. 2006;59(3):228-235.

28. Grassi G, Seravalle G, Calhoun DA, et al. Mechanisms responsible for sympathetic activation by cigarette smoking in humans. Circulation. 1994; 90(1):248-253.

29. Mårin P, Darin N, Amemiya T, et al. Cortisol secretion in relation to body fat distribution in obese premenopausal women. Metabolism. 1992;41(8):882-886.

30. Bajaj M. Nicotine and Insulin Resistance: When the Smoke Clears. Diabetes. 2012;61(12):3078-3080.

31. Targher G, Alberiche M, Zenere MB, et al. Cigarette smoking and insulin resistance in patients with noninsulin-dependent diabetes mellitus. The Journal of Clinical Endocrinology \& Metabolism. 1997; 82(11):36193624.

32. Baron JA, La Vecchia C, Levi F. The antiestrogenic effect of cigarette smoking in women. American journal of obstetrics and gynecology. 1990;162(2):502-514.
33. Clair C, Chiolero A, Faeh D, et al. Dose-dependent positive association between cigarette smoking, abdominal obesity and body fat: crosssectional data from a population-based survey. BMC Public Health. 2011;11:23.

34. Brook JS, Zhang C, Seltzer N, et al. Insomnia in Adults: The Impact of Earlier Cigarette Smoking from Adolescence to Adulthood. Journal of addiction medicine. 2015; 9(1):40-45.

35. Hughes JR. Smoking and Suicide: A Brief Overview. Drug and alcohol dependence. 2009;98(3):169-178.

36. Shaikh RA, Siahpush M, Singh GK, et al. Socioeconomic Status, Smoking, Alcohol use, Physical Activity, and Dietary Behavior as Determinants of Obesity and Body Mass Index in the United States: Findings from the National Health Interview Survey. International journal of $\mathrm{MCH}$ and AIDS. 2015;4(1):22-34

37. Albanes D, Jones DY, Micozzi MS, et al. Associations between smoking and body weight in the US population: analysis of NHANES II. American Journal of Public Health. 1987;77(4):439-444. 